Cochrane Database of Systematic Reviews

\title{
Interventions for the treatment of persistent post-COVID-19 olfactory dysfunction (Protocol)
}

Webster KE, MacKeith S, Philpott C, Hopkins C, Burton MJ

Webster KE, MacKeith S, Philpott C, Hopkins C, Burton MJ.

Interventions for the treatment of persistent post-COVID-19 olfactory dysfunction (Protocol).

Cochrane Database of Systematic Reviews 2021, Issue 2. Art. No.: CD013876.

DOI: 10.1002/14651858.CD013876.

www.cochranelibrary.com 
TABLE OF CONTENTS

HEADER 1

ABSTRACT

BACKGROUND

OBJECTIVES

METHODS

ACKNOWLEDGEMENTS

REFERENCES

APPENDICES

HISTORY

CONTRIBUTIONS OF AUTHORS

DECLARATIONS OF INTEREST

SOURCES OF SUPPORT 
[Intervention Protocol]

\section{Interventions for the treatment of persistent post-COVID-19 olfactory dysfunction}

Katie E Webster ${ }^{1}$, Samuel MacKeith ${ }^{2}$, Carl Philpott ${ }^{3}$, Claire Hopkins ${ }^{4}$, Martin J Burton 5

${ }^{1}$ Cochrane ENT, Nuffield Department of Surgical Sciences, University of Oxford, Oxford, UK. 2Oxford University Hospitals NHS Foundation Trust, Oxford, UK. ${ }^{3}$ Department of Medicine, Norwich Medical School, University of East Anglia, Norwich, UK. ${ }^{4}$ ENT Department, Guy's Hospital, London, UK. ${ }^{5}$ Cochrane UK, Oxford, UK

Contact address: Katie E Webster, katie.webster@doctors.org.uk.

Editorial group: Cochrane ENT Group.

Publication status and date: New, published in Issue 2, 2021.

Citation: Webster KE, MacKeith S, Philpott C, Hopkins C, Burton MJ. Interventions for the treatment of persistent postCOVID-19 olfactory dysfunction (Protocol). Cochrane Database of Systematic Reviews 2021, Issue 2. Art. No.: CD013876. DOI: 10.1002/14651858.CD013876.

Copyright @ 2021 The Cochrane Collaboration. Published by John Wiley \& Sons, Ltd.

\section{A B S T R A C T}

\section{Objectives}

This is a protocol for a Cochrane Review (intervention). The objectives are as follows:

To assess the effects (benefits and harms) of interventions to treat olfactory dysfunction in people with COVID-19 infection.

A secondary objective is to maintain the currency of the evidence, using a living systematic review approach. 


\section{B A C K G R O U N D}

\section{Description of the condition}

Loss of olfactory function (the sense of smell) as a consequence of COVID-19 infection was first recognised in March 2020 (Hopkins 2020a). Since that time, it has become established that this is a cardinal symptom of COVID-19 infection (Menni 2020), with a high predictive value (Gerkin 2020). This usually takes the form of complete or partial loss of olfactory function (anosmia and hyposmia respectively) (Lechien 2020).

Olfactory dysfunction, through loss (quantitative changes) or distortion (qualitative changes) of smell, is a debilitating condition with a variety of causes and has a major impact on quality of life (Croy 2014; Erskine 2020; Philpott 2014). It also has safety implications, through the inability to detect odours that may signal danger (such as smoke, gas or spoilt food). Post-infectious olfactory dysfunction (PIOD) is one of the most common causes of olfactory dysfunction, representing up to $20 \%$ of all cases in specialist olfactory clinics (Cain 1988; Damm 2004; Seiden 2001). Many viruses have been implicated in PIOD, including the coronavirus family. However, the prominence of SARS-CoV-2 (which causes COVID-19) as a causative agent has been notable, and can perhaps be attributed to the spotlight created by it being the cause of a pandemic.

Accurate estimates of the prevalence of olfactory dysfunction resulting from COVID-19 are difficult to obtain, and may vary according to the clinical presentation of the disease (which ranges from mild, or relatively asymptomatic, to serious complications requiring intensive care). A recent systematic review identified an overall prevalence of smell loss of $43 \%$, however the authors noted high variation between the estimates from different studies (von Bartheld 2020). Another systematic review showed a prevalence of $62 \%$ across the range of studies included (Rocke 2020). A large European cohort, which included hospitalised individuals with mild-moderate symptoms, as well as individuals who did not require hospital treatment, reported the prevalence of olfactory dysfunction to be $85.6 \%$ (Lechien 2020). The majority of individuals included in this study reported anosmia, with a minority reporting hyposmia (20.4\%).

The incidence of anosmia or olfactory dysfunction related to COVID-19 appears to vary across the world, with studies from the USA and Europe typically demonstrating much higher incidence than those from Asia (Meng 2020; von Bartheld 2020). A study from Wuhan, China, reported abnormalities of olfactory function in only $5.1 \%$ of their cohort (214 patients, with both severe and mild forms of the disease) (Mao 2020). It is not clear why this may be. Gender and age have also been suggested as possible effect modifiers, with some reviews suggesting preponderance in females (Meng 2020), and others suggesting an increased incidence in younger age groups (Fuccillo 2020).

The incidence of olfactory dysfunction may also vary depending on the method used to diagnose it. Studies that used self-reported symptoms of loss of smell identified a lower prevalence than those that utilised some form of objective assessment (von Bartheld 2020). It is well recognised that, for healthy individuals, self-rating of the sense of smell may correlate poorly with scores achieved on psychophysical testing (Landis 2003). Correlation is better for those who report olfactory dysfunction (particularly anosmia), but on an individual level there is still considerable variation between the severity of the reported loss, and that identified with psychophysical tests (Welge-Luessen 2005). With larger numbers reporting COVID-19 symptoms in general, the data collected by the COVID tracker app is more likely to reflect the prevalence of olfactory dysfunction in the non-hospitalised population (Menni 2020).

A further complication in obtaining accurate estimates of prevalence is the variety of data sources that are available. Studies conducted in a hospitalised population may present very different estimates to those where data are gathered from internet-based surveys. This may reflect genuine differences in the presence of olfactory dysfunction in these varied populations, different methods of ascertaining olfactory function, or potentially a different preponderance to report symptoms. Internet-based surveys may have a greater propensity for responder bias than other cross-sectional studies - those who have symptoms may be more likely to participate or complete the required data, resulting in inflated estimates of prevalence. However, some prospective series have also identified a high prevalence of olfactory dysfunction (Spinato 2020).

Other symptoms of olfactory dysfunction include phantosmia (qualitative dysfunction in the absence of an odour, or 'olfactory hallucinations') and parosmia (distorted perception of an odour stimulus) (Hummel 2016). A recent survey of individuals with COVID-19 indicated that these symptoms occurred in fewer than $10 \%$ in the short term (Parma 2020). However, longer-term followup may demonstrate further problems at a later stage, and reports of persisting parosmia as a consequence of COVID-19 are increasing (Hopkins 2020b).

The exact mechanism by which the SARS-CoV-2 virus triggers olfactory dysfunction remains unclear (reviewed in Butowt 2020). Many viruses cause conductive olfactory impairment, with inflammation, nasal congestion and rhinorrhoea preventing detection of odours during the acute phase of the infection. These symptoms are not as common in COVID-19 and, when present, do not correlate well with the degree of olfactory dysfunction (Parma 2020). Symptoms may also be caused by direct damage to, or death of, olfactory neurons or cells within the olfactory bulb. However, olfactory neurons lack ACE2 receptors (which facilitate viral entry to cells) and the rapid recovery for most individuals with COVID-19 related smell loss makes this less likely. Infection of supporting cells (sustentacular cells) within the olfactory epithelium has been reported (reviewed in Bilinska 2020). These cells play a critical role in supporting the function of olfactory neurons, and their infection may consequently have an adverse effect on olfactory processing.

For many individuals with COVID-19 related olfactory dysfunction, the condition is temporary, and they recover a normal sense of smell relatively quickly (Chary 2020; Klopfenstein 2020). Complete recovery by two weeks was reported for most people $(96.7 \%)$ in the study by Lechien 2020. A second case series of individuals with mild coronavirus symptoms found that $89 \%$ had complete or partial recovery of olfactory function by four weeks from the onset of the disease (Boscolo-Rizzo 2020). However, for some individuals the problem persists. Some studies report a much higher prevalence of persisting olfactory loss, despite resolution of other COVID-19 symptoms. Data from the Global Consortium of Chemosensory Research indicates that up to $50.7 \%$ of individuals may have persisting olfactory dysfunction at up to 40 days from 
the onset of COVID-19 (Gerkin 2020). It remains unclear why some individuals experience longer lasting olfactory deficits. This may be due to differing extents of damage (as suggested by Butowt 2020), or different mechanisms for olfactory loss (Hopkins 2020c; Saussez 2020). Differing features of COVID-19 related smell loss may include a potential impact on true gustatory function, as well as a greater severity of olfactory loss itself (Huart 2020); many larger studies are limited by the reliance on self-reporting, so this is more difficult to corroborate.

This review is one of a pair that consider the effects of interventions to prevent or treat persisting olfactory dysfunction following COVID-19. For this review, we consider treatment for individuals who already have persisting olfactory dysfunction at four weeks (or longer) following a diagnosis of COVID-19. For the companion review ('Interventions for the prevention of persisting olfactory dysfunction following COVID-19'; Webster 2021), we consider interventions that may be used in the acute phase (less than four weeks since diagnosis), aiming to prevent individuals from developing persisting olfactory dysfunction.

\section{Description of the intervention}

As COVID-19 related persistent olfactory dysfunction is a relatively new condition, there are no established treatments for it. However, a number of interventions have been used for other, post-viral, causes of anosmia. Steroids are commonly prescribed for olfactory dysfunction - these are typically administered locally as a nasal spray, drops or rinse for conductive causes of olfactory loss - where the nasal cavity is blocked, or partially blocked, by inflammation and oedema. Systemic (oral) steroids may also be used, particularly in cases where no conductive cause is identified.

Olfactory training is also frequently suggested for reduced or absent sense of smell - this involves regular exposure to a number of specific odours. It can be performed in a variety of different ways, using household items or essential oils.

A large number of other interventions have been used for PIOD, and may therefore be of use for post-COVID-19 olfactory dysfunction. A variety of vitamins, minerals and nutritional supplements have been proposed to be of benefit - either taken as an oral supplement or, in some instances, used intranasally (such as intranasal vitamin A drops). Glutamate antagonists and xanthine derivatives are used occasionally in the treatment of post-viral olfactory dysfunction and may therefore be assessed in relation to COVID-19. Trials of acupuncture have also taken place.

Olfactory dysfunction has a considerable impact on quality of life and may be a long lasting or even permanent condition. Psychological therapies, such as counselling or cognitive behavioural therapy, may therefore help to develop coping mechanisms and improve quality of life, even in the absence of objective improvement in the sense of smell.

Clinical trials are ongoing to assess a variety of interventions for the treatment of COVID-19. These include antivirals, such as remdesivir, and monoclonal antibodies. It is possible that these interventions may also benefit individuals with olfactory dysfunction, if these symptoms are assessed.

For many individuals, smell loss is anticipated to improve with time. There is no intervention that could currently be regarded as standard care for individuals with post-COVID-19 related anosmia.
Interventions are therefore likely to be compared to no treatment, or to placebo (dummy) treatment. However, olfactory training is often suggested as an intervention with few, if any, adverse effects, and may be used alongside other treatments, therefore we anticipate that this may be advised to be undertaken concurrently in some studies.

\section{How the intervention might work}

Steroids are frequently prescribed to ensure that any intranasal inflammatory component that is exacerbating the PIOD is adequately treated. Whether steroids have a persisting effect after discontinuation is unclear. Intranasal steroids are used for a number of other conditions, and serious side effects are rare, but they may cause nasal irritation, nosebleeds or other localised complications. Steroids may also be administered systemically typically as oral tablets, or sometimes parenterally.

Olfactory training aims to stimulate the olfactory neurons with a variety of odours in order to enhance smell detection. It is unclear whether any changes occur within the olfactory epithelium itself, in the olfactory bulb, or involve reorganisation of neural olfactory pathways. Although olfactory training may not restore olfactory function, it may improve the performance of the olfactory system. Two recent reviews suggest that olfactory training may give some benefit to those with olfactory disorders (Pekala 2016; Sorokowska 2017). However, the majority of included studies were prospective cohorts, with only one RCT included.

A number of vitamins and minerals have been suggested to have a beneficial effect on the olfactory epithelium, including vitamins A, B12 and D, and zinc. It is thought that metabolites of vitamin A may play a role in regeneration of tissue in the olfactory epithelium or olfactory bulb, and this has been used intranasally to treat individuals with post-viral olfactory loss (Hummel 2017). Vitamin B12 is known to be important in the maintenance of central and peripheral nervous function, and deficiency of vitamin B12 has been associated with olfactory impairment (Derin 2016). Vitamin D deficiency has also been linked to olfactory impairment (Bigman 2020), and there is ongoing interest in the potential use of vitamin D to prevent or treat other symptoms of COVID-19 infection (Martineau 2020). Zinc deficiency has also been shown to have an association with olfactory dysfunction and zinc was historically used intranasally as a potential treatment for anosmia, although there are concerns over toxicity (Alexander 2006).

Antioxidants, such as alpha lipoic acid and omega 3 fatty acids, have also been suggested as possible interventions to treat anosmia (Hummel 2002). They are thought to have neuroprotective properties that may help restore function within olfactory neurons or the olfactory bulb. Minocycline has also been trialled in post-viral olfactory loss - due to its neuroprotective properties, rather than its traditional role as an antibiotic (Reden 2011).

The impact of olfactory dysfunction on quality of life is substantial. Adjusting to, and learning to cope with, this life-changing symptom may be helped through psychological therapies, counselling or cognitive behavioural therapy.

It is possible that antiviral agents, some of which have already been shown to impact on the severity of COVID-19, may also affect the olfactory dysfunction. Reducing viral replication (and consequently lowering the viral load in an individual) may result in reduced 
severity of olfactory loss, or hasten the recovery. Monoclonal antibodies have also been used to treat COVID-19, and could also have an impact on the severity and persistence of olfactory impairment.

There have also been small studies to assess the possible benefit of acupuncture in olfactory loss (Dai 2016; Vent 2010).

Glutamate plays an important role in neurotransmission for olfactory neurons and within the olfactory bulb. Glutamate antagonists, such as caroverine, have been proposed to help protect against neurotoxicity, and consequently improve olfactory function (Quint 2002). Finally, xanthine derivatives such as theophylline and pentoxifylline have been proposed to stimulate olfactory neuron activity, and may therefore have an effect on olfactory function.

It is possible that individuals with a longer duration of anosmia have a different underlying disease process than those with temporary olfactory dysfunction related to COVID-19. Consequently, the efficacy of different interventions may vary between these groups.

\section{Why it is important to do this review}

The COVID-19 pandemic has resulted in an enormous number of individuals becoming infected with SARS-CoV-2. Fortunately, many individuals recover completely. However, the long-term consequences of infection are only just becoming apparent. Although the prevalence of persisting olfactory dysfunction may be small, with huge numbers of global infections the actual number of individuals suffering from post-COVID-19 related persistent anosmia is large. We can assume an estimated $60 \%$ suffer olfactory dysfunction at the onset of the infection and that at least $10 \%$ of these go on to experience PIOD. Given the number of infections (> 50 million infections worldwide, as of November 2020), we estimate that up to three million people may have been affected to date. The burden of this disorder is also considerable, with significant effects on quality of life, as well as safety implications (due to the inability to detect harmful or dangerous smells). Therefore, identification of potential treatments that may improve the outcome for sufferers is timely and important.

Many interventions carry a risk of adverse effects. If the beneficial effect of treatment is small or negligible, then side effects may be such that individuals do not consider treatments worthwhile. With this review we aim to comprehensively assess the benefits and harms of interventions to treat post-COVID-19 related olfactory dysfunction, to ensure that patients can make an informed choice regarding the management of their condition.

Given the recent emergence of COVID-19, there is currently a great deal of uncertainty about how best to manage the olfactory dysfunction that occurs as a result of the virus. The sheer number of infected individuals worldwide also means that evidence that supports decision making for the management of COVID-19 is a priority for decision makers globally. There is also a strong emphasis on COVID-19 research at present, therefore we anticipate that there is likely to be new evidence available over the coming months and years. Therefore, this review will be a living systematic review, which will be continually updated to incorporate any important new evidence as it becomes available.

\section{O B JECTIVES}

To assess the effects (benefits and harms) of interventions to treat olfactory dysfunction in people with COVID-19 infection.

A secondary objective is to maintain the currency of the evidence, using a living systematic review approach.

\section{METHODS}

\section{Criteria for considering studies for this review}

\section{Types of studies}

We will include randomised controlled trials and quasi-randomised trials (where trials were designed as RCTs, but the sequence generation for allocation of treatment used methods such as alternative allocation, birth dates, alphabetical order etc.).

We considered that olfactory dysfunction is unlikely to be stable over long periods of time, and individuals may experience considerable fluctuation of symptoms over a given time period. Therefore, cross-over trials are unlikely to be identified in this area. If we do identify any cross-over studies, we will only include data from the first phase of these studies in the review.

We will only include studies where patients were followed up for at least one week. The aim of this review is to synthesise evidence for treatments that may have a lasting effect on olfactory function, rather than those that may have a very brief or temporary impact.

We will include studies regardless of their publication status or language of publication. We will include outcome data reported on a trial registry, even if no published results are available. If we identify material from a pre-print server then we will initially note this in the 'What's new' section of the review, pending the identification of fully published data. If no published data are identified within four months of the pre-print article being made available then the data will be incorporated in the review.

\section{Types of participants}

Adult participants (aged 18 years or older) with persisting abnormalities of their sense of smell as a consequence of COVID-19. For the purpose of this review, the term 'persisting' refers to olfactory dysfunction being present at four weeks following a diagnosis of COVID-19. We anticipate that some studies will report this as four weeks of olfactory dysfunction, rather than four weeks since a positive test for COVID-19 - either of these measures will be included in the review.

We will include individuals with anosmia (absent sense of smell) or hyposmia (reduced sense of smell). We anticipate that some trials may also include a small number of individuals with symptoms of pure parosmia or phantosmia. We will include data from these trials, providing the majority of participants $(\geq 80 \%)$ report anosmia or hyposmia.

We will include studies where olfactory dysfunction has been identified with either psychophysical (objective) testing, or through self-report of symptoms. We will investigate whether this has any impact on the effect estimates using subgroup analysis (see Subgroup analysis and investigation of heterogeneity). 
We will include studies where COVID-19 has been diagnosed through either objective testing (e.g. viral polymerase chain reaction (PCR) from nasopharyngeal swabs) or through a clinical diagnosis (for example, sudden onset of olfactory dysfunction with other symptoms of COVID-19, or in the context of contact with an infected individual).

For inclusion in this review, all participants in the trial must have abnormalities of their sense of smell. We will not include studies where only some participants are eligible (i.e. not all participants had olfactory dysfunction at the start of the trial).

\section{Types of interventions}

\section{Interventions}

We will include any intervention proposed specifically to treat olfactory disturbance. We anticipate that this may include, but is not limited to, the following interventions:

- Intranasal steroid drops/rinses

- Intranasal steroid sprays

- Systemic steroids

- Olfactory training

- Intranasal vitamin A

- Zinc

- Antioxidants (e.g. omega 3 fatty acids, alpha lipoic acid)

- Counselling

- Antiviral agents (e.g. remdesivir)

- Other antimicrobials (e.g. minocycline)

- Other vitamins and nutritional supplements (to be analysed according to the type of vitamin/supplement, rather than as a pooled comparison)

- Acupuncture

- Monoclonal antibodies

- Glutamate antagonists (e.g. caroverine)

- Xanthine derivatives (e.g. theophylline, pentoxifylline)

All routes of administration, doses and duration of treatment will be included.

We will exclude studies that consider surgery, as this is not currently an intervention of interest for post-viral olfactory loss.

Olfactory training is considered to be a complex intervention, as the method of delivery may vary considerably in different studies. This will be assessed using subgroup analyses (see below).

\section{Comparator(s)}

The main comparison will be:

- placebo or no treatment.

\section{Concurrent treatments}

We anticipate that some trials may include olfactory training (or other interventions) as concurrent therapy for both arms. There will be no limits on the type of concurrent treatments used. We will pool these trials with studies where no concurrent treatment was used and we will use sensitivity analyses to determine whether the effect estimates are changed because of this.

\section{Types of outcome measures}

We will analyse the following outcomes in the review, but we will not use them as a basis for including or excluding studies. Where possible, all outcomes will be reported at three time points:

- 1 to 3 months (this is the main time point of interest);

- > 3 months to 12 months;

- > 12 months to 3 years.

\section{Primary outcomes}

- Recovery of sense of smell:

* as assessed by the participants;

* as assessed with psychophysical testing, using Sniffin' Sticks, University of Pennsylvania Smell Identification Test (UPSIT) or another validated test.

- Disease-related quality of life, as assessed by the Olfactory Disorders Questionnaire, or another validated questionnaire (which specifically relates to olfactory dysfunction).

- Serious adverse effects (as defined by the trialists).

\section{Secondary outcomes}

- Change in sense of smell:

* as assessed by the participants;

* as assessed by psychophysical testing, using Sniffin' Sticks, UPSIT or another validated test.

- Overall, generic quality of life, as assessed by validated methods (e.g. EQ-5D).

- Presence of parosmia, as reported by the participants.

- Other adverse effects (including nosebleeds/bloody discharge).

Where possible, we will compare the threshold for appreciable change in these outcomes to published minimally important differences (MID). These have been reported for psychophysical olfactory testing using Sniffin' Sticks (MID 5.5 points, Gudziol 2006) and the Olfactory Disorders Questionnaire (MID 5.2 points, Mattos 2018).

\section{Search methods for identification of studies}

The Cochrane ENT Information Specialist will conduct systematic searches for randomised controlled trials and controlled clinical trials. There will be no language or publication status restrictions. Some of the search terms will be limited by publication year, due to the novel nature of post-COVID-19 olfactory dysfunction. We may contact original authors for clarification and further data if trial reports are unclear and we will arrange translations of papers where necessary.

\section{Electronic searches}

Published, unpublished and ongoing studies will be identified by searching the following databases from their inception:

- the Cochrane ENT Trials Register (search via the Cochrane Register of Studies to date);

- the Cochrane Central Register of Controlled Trials (CENTRAL) (search via the Cochrane Register of Studies to date);

- Ovid MEDLINE(R) Epub Ahead of Print, In-Process \& Other NonIndexed Citations, Ovid MEDLINE(R) Daily and Ovid MEDLINE(R) (1946 to date); 
- Ovid Embase (1974 to date);

- Web of Knowledge, Web of Science (1945 to date);

- ClinicalTrials.gov, www.clinicaltrials.gov:

* search via the Cochrane Register of Studies to date;

* search via www.clinicaltrials.gov to date;

- World Health Organization (WHO) International Clinical Trials Registry Platform (ICTRP):

* search via the Cochrane Register of Studies to date;

* search via https://apps.who.int/trialsearch/ to date);

- Cochrane COVID-19 Study Register, https:// covid-19.cochrane.org/ (search via the Cochrane Register to date);

- World Health Organization (WHO) COVID-19 'Global literature on coronavirus disease', https://search.bvsalud.org/globalliterature-on-novel-coronavirus-2019-ncov (search to date).

The subject strategies for databases will be modelled on the draft search strategies in Appendix 1. Where appropriate, these will be combined with subject strategy adaptations of the highly sensitive search strategy designed by Cochrane for identifying randomised controlled trials and controlled clinical trials (as described in the Technical Supplement to Chapter 4 of the Cochrane Handbook for Systematic Reviews of Interventions version 6.1) (Lefebvre 2020).

Clinical trials are ongoing to assess a variety of interventions for the treatment of COVID-19. As few studies have currently been published, the search strategy developed is highly sensitive in order to try to capture all interventions as they are introduced. The Information Specialist will review the search methods (the sources and search frequency) and the search terms (index terms and free text terms) on an annual basis. The search strategy may evolve over time, as a greater body of literature is published and a more focused list of interventions are identified.

\section{Living systematic review considerations}

As a living systematic review, the Information Specialist will conduct monthly searches of the sources listed above, except the following, which will be searched less frequently and, as a minimum, on a:

\section{- quarterly basis:}

* World Health Organization (WHO) COVID-19 'Global literature on coronavirus disease' https://search.bvsalud.org/globalliterature-on-novel-coronavirus-2019-ncov (search to date);

* COAP COVID-19 Living Evidence, Institute of Social and Preventive Medicine (ISPM), University of Bern https:// zika.ispm.unibe.ch/assets/data/pub/search_beta/ (search to date); or

- an annual basis:

* ClinicalTrials.gov (search via www.clinicaltrials.gov to date);

* World Health Organization (WHO) International Clinical Trials Registry Platform (ICTRP) (search via https://apps.who.int/ trialsearch/to date).

Clinical trials are ongoing to assess a variety of interventions for the treatment of COVID-19. We plan to conduct surveillance activity and commence monthly searches when we anticipate that the first trials will have data available.
The Information Specialist will apply appropriate date restrictions and auto alerts as available and appropriate, and will provide details in an appendix to the published review.

\section{Searching other resources}

We will scan the reference lists of identified publications for additional trials and contact trial authors if necessary. The Information Specialist will also run non-systematic searches of Google Scholar to retrieve grey literature and other sources of potential trials.

We will not perform a separate search for adverse effects. We will consider adverse effects described in included studies only.

We will make efforts to identify full-text papers regardless of language of publication and endeavour to seek help with translation; however, we will not hold up the rapid review process. Any papers that we are unable to source in time for the scheduled living review update or are unable to get translated will be listed as awaiting assessment.

\section{Living systematic review considerations}

As a living systematic review, we will scan the reference lists of identified publications for additional trials and contact trial authors if necessary. In addition, the Information Specialist will search on an annual basis Ovid MEDLINE to retrieve existing systematic reviews relevant to this systematic review, so that we can scan their reference lists for additional trials. The Information Specialist will conduct annual searches of the Web Knowledge Science Citation Index for articles referencing the published review and its included studies and carry out non-systematic searches of Google Scholar to retrieve grey literature and other sources of potential trials.

\section{Data collection and analysis}

\section{Selection of studies}

We will consider using Cochrane's Screen4Me workflow to help assess the search results, depending on the number of results retrieved from the database searches. Screen4Me comprises three components:

1. Known assessments - a service that matches records in the search results to records that have already been screened in Cochrane Crowd and been labelled as 'a RCT' or as 'not a RCT'.

2. The machine learning classifier (RCT model) (Wallace 2017), available in the Cochrane Register of Studies (CRS-Web), which assigns a probability of being a true RCT (from 0 to 100) to each citation. For citations that are assigned a probability score below the cut-point at a recall of $99 \%$ we will assume these to be non-RCTs. For those that score on or above the cut-point we will either manually dual screen these results or send them to Cochrane Crowd for screening.

3. Cochrane Crowd is Cochrane's citizen science platform where the Crowd help to identify and describe health evidence. For more information about Screen4Me and the evaluations that have been done, please go to the Screen4Me website on the Cochrane Information Specialist's portal and see Marshall 2018, McDonald 2017, Noel-Storr 2018 and Thomas 2017.

At least two review authors will independently screen the remaining titles and abstracts to identify potentially relevant studies. At least two review authors will independently evaluate 
the full text of each potentially relevant study to determine whether it meets the inclusion/exclusion criteria for this review. Any differences will be resolved by discussion and consensus, with the involvement of a third author where necessary.

\section{Living systematic review considerations}

We will immediately screen any new citations retrieved by the monthly searches using the approach outlined above.

\section{Data extraction and management}

At least two review authors will independently extract outcome data from each study using a standardised data collection form. Where a study has more than one publication, we will retrieve all publications to ensure complete extraction of data. Any discrepancies in the data extracted by the two authors will be checked against the original reports, and differences will be resolved through discussion and consensus, with recourse to a third author where necessary. If required, we will contact the study authors for clarification.

We will collect information on study design and setting, participant characteristics (including disease severity and age), study eligibility criteria, details of the intervention(s) given, the outcomes assessed, the source of study funding and any conflicts of interest stated by the investigators. We will also include details of the baseline characteristics of trial participants, with particular regard to prognostic features such as age, gender, severity of infection and duration of time since COVID-19 infection.

The primary effect of interest for this review will be the effect of treatment assignment (which reflects the outcomes of treatment for people who were assigned to the intervention) rather than a per protocol analysis (the outcomes of treatment only for those who completed the full course of treatment as planned). For the outcomes of interest in this review, we will extract the findings from the studies on an available case basis, i.e. all available data from all participants at each time point, based on the treatment to which they were randomised. This will be irrespective of compliance, or whether participants had received the intervention as planned.

In addition to extracting pre-specified information about study characteristics and aspects of methodology relevant to risk of bias, we will extract the following summary statistics for each trial and outcome:

- For continuous data: the mean values, standard deviation and number of patients for each treatment group at the different time points for outcome measurement. Where endpoint data are not available, we will extract the values for change-frombaseline data instead. If values for the individual treatment groups are not reported, where possible we will extract summary statistics (e.g. mean difference) from the studies.

- For binary data: we will extract information on the number of participants experiencing an event, and the number of participants assessed at that time point. If values for the individual treatment groups are not reported, where possible we will extract summary statistics (e.g. risk ratio) from the studies.

- For ordinal scale data: if we identify data reported on an ordinal scale and if the data appear to be normally distributed, or if the analysis performed by the investigators indicates that parametric tests are appropriate, then we will treat the outcome measure as continuous data. Alternatively, if data are available, we will convert these to binary data.

- For time-to-event data: if we identify data reported as time-toevent then, where possible, we will extract data on hazard ratios from individual studies. If these data are not provided then we will extract alternative measures of treatment effect, such as the observed and expected number of events in each group, a $\mathrm{P}$ value and the number of events in each arm, or data in a Kaplan Meier curve.

We have pre-specified time points of interest for the outcomes in this review. Where studies report data at multiple time points, we will take the longest available follow-up point within each of the specific time frames. For example, if a study reports an outcome at 4 weeks, 8 weeks and 12 weeks of follow-up then the 12-week data will be included for the time point 1 to 3 months.

\section{Assessment of risk of bias in included studies}

Two authors will undertake assessment of the risk of bias of the included trials independently, with the following taken into consideration, as guided by the Cochrane Handbook for Systematic Reviews of Interventions (Handbook 2011):

- sequence generation;

- allocation concealment;

- blinding;

- incomplete outcome data;

- selective outcome reporting; and

- other sources of bias.

We will use the Cochrane 'Risk of bias' tool in RevMan 5.4 (RevMan 2020), which involves describing each of these domains as reported in the trial and then assigning a judgement about the adequacy of each entry: 'low', 'high' or 'unclear' risk of bias. Any discrepancies in judgement between the two authors will be resolved through discussion, and with recourse to a third author where necessary.

\section{Measures of treatment effect}

We will summarise the effects of dichotomous outcomes (e.g. recovery of sense of smell) as risk ratios (RR) with 95\% confidence intervals $(\mathrm{Cls})$. For the key outcomes that we will present in the 'Summary of findings' tables, we will also express the results as absolute numbers based on the pooled results and compared to the assumed risk. We may also calculate the number needed to treat to benefit (NNTB) using the pooled results. The assumed baseline risk will be either (a) the median of the risks of the control groups in the included studies - this being used to represent a 'medium-risk population' or, alternatively, (b) the average risk of the control groups in the included studies - used as the 'study population' (Handbook 2020). If a large number of studies are available (and where appropriate) we may also present additional data based on the assumed baseline risk in (c) a low-risk population and (d) a high-risk population.

For continuous outcomes, we will express treatment effects as a mean difference (MD) with standard deviation (SD) or as a standardised mean difference (SMD) if different scales have been used to measure the same outcome. We will provide a clinical interpretation of the SMD values using either Cohen's $d$ or by conversion to a recognised scale if possible. 
For time-to-event outcomes we will summarise the effects as a hazard ratio (HR) with $95 \% \mathrm{Cl}$. If necessary, and where possible (if sufficient alternative data are provided), we will estimate the HR from individual studies according to the methods outlined in Tierney 2007.

\section{Unit of analysis issues}

Cross-over trials and cluster-randomised trials are not anticipated for this review topic. Post-COVID-19 related anosmia is unlikely to be a stable condition, and interventions may not have a temporary effect. If cross-over trials are identified then we plan to use only the data from the first phase of the study. If cluster-randomised trials are identified then we will ensure that analysis methods are used to account for clustering in the data (Handbook 2020).

If we identify multi-arm trials for inclusion in the review, we will ensure that multiple intervention groups are analysed in an appropriate way to avoid arbitrary omission of groups or double counting of participants. This may include combining intervention groups (if appropriate) or splitting the 'shared' group into two or more groups with a smaller sample size.

\section{Dealing with missing data}

We will try to contact study authors via email whenever the outcome of interest is not reported, if the methods of the study suggest that the outcome had been measured. We will do the same if not all data required for meta-analysis have been reported, unless the missing data are standard deviations. If standard deviation data are not available, we will approximate these using the standard estimation methods from $\mathrm{P}$ values, standard errors or $95 \% \mathrm{Cls}$ if these are reported as detailed in the Cochrane Handbook for Systematic Reviews of Interventions (Handbook 2020). If it is impossible to estimate these, we will contact the study authors.

Apart from imputations for missing standard deviations, we will conduct no other imputations. We will extract and analyse all data using the available case analysis method.

\section{Assessment of heterogeneity}

We will assess clinical heterogeneity (which may be present even in the absence of statistical heterogeneity) by examining the included trials for potential differences between studies in the types of participants recruited, interventions or controls used and the outcomes measured.

We will assess statistical heterogeneity by visually inspecting the forest plots and by considering the $\mathrm{Chi}^{2}$ test (with a significance level set at $P$ value $<0.10$ ) and the $I^{2}$ statistic, which calculates the percentage of variability that is due to heterogeneity rather than chance (Handbook 2020).

\section{Assessment of reporting biases}

We will assess reporting bias as within-study outcome reporting bias and between-study publication bias.

\section{Outcome reporting bias (within-study reporting bias)}

We will assess within-study reporting bias by comparing the outcomes reported in the published report against the study protocol or trial registry, whenever this can be obtained. If the protocol or trial registry entry is not available, we will compare the outcomes reported to those listed in the methods section. If results are mentioned but not reported adequately in a way that allows analysis (e.g. the report only mentions whether the results were statistically significant or not), bias in a meta-analysis is likely to occur. We will seek further information from the study authors. If no further information can be found, we will note this as being a 'high' risk of bias when the 'Risk of bias' tool is used. If there is insufficient information to judge the risk of bias we will note this as an 'unclear' risk of bias (Handbook 2011).

\section{Publication bias (between-study reporting bias)}

We will assess funnel plots if sufficient studies (more than 10) are available for an outcome. If we observe asymmetry of the funnel plot, we will conduct more formal investigation using the methods proposed by Egger 1997. We will also report on whether there were any studies identified through trial registries and other sources (Searching other resources) with unpublished reports.

\section{Data synthesis}

Where possible and appropriate (if participants, interventions, comparisons and outcomes are sufficiently similar in the trials identified) we will conduct a quantitative synthesis of results. We will conduct all meta-analyses using a fixed-effect method in RevMan 5.4.

We will include all studies in the meta-analyses, regardless of their risk of bias. However, we will incorporate a summary assessment of risk of bias in the measure of certainty of the evidence for each outcome, using the GRADE system.

For dichotomous data, we plan to analyse treatment differences as a risk ratio (RR) calculated using the fixed-effect Mantel-Haenszel methods.

For continuous outcomes, we will use the inverse variance, fixedeffect method of meta-analysis. If all data are from the same scale, we will pool mean follow-up values with change-from-baseline data and report this as a mean difference. If there is a need to report standardised mean differences then we will not pool endpoint and change-from-baseline data.

For time-to-event data we plan to use a generic inverse variance, fixed-effect method of meta-analysis.

Sense of smell may be tested using a variety of methods, which consider different aspects of the sense of smell. These are:

- identification - the ability to identify and name a specific odour; - threshold - the concentration of an odour that can be detected; - discrimination - the ability to discriminate between odours.

We will include methods that consider any or all of the above aspects of sense of smell. Where meta-analysis is appropriate, we will only pool results that look at the same individual aspect (or aspects) of sense of smell.

If meta-analysis is not possible (for example, due to incompletely reported outcomes/effect estimates or different effect measures that cannot be combined) then we will consider presenting alternative synthesis methods. This may include summarising the effect estimates from individual studies, combining P values or vote counting based on the direction of effect, depending on the data available. 


\section{Living systematic review considerations}

Whenever new evidence relevant to the review is identified in our monthly searches, we will extract the data, assess risk of bias and incorporate it into the synthesis every four months, as appropriate. Formal sequential meta-analysis approaches will not be used for updated meta-analyses.

\section{Subgroup analysis and investigation of heterogeneity}

A number of factors are likely to impact on the outcomes included in this review. Where possible (if appropriate data are reported), we will assess these with subgroup analysis, regardless of whether statistical heterogeneity is identified. These are the following:

- Age of participants in the trial (under 60 years versus those aged 60 or over):

* age is well recognised to impact on olfactory function, with sense of smell worsening with time. The ability to detect smells may therefore differ considerably between younger and older adults.

- Gender of participants in the trial (female versus male):

* gender has an influence on olfactory function and may also impact recovery rates.

- Method used to determine olfactory dysfunction at trial baseline (self-reported versus psychophysical testing):

* rates of olfactory dysfunction vary depending on whether self-report or psychophysical testing is used to identify olfactory loss. Effect estimates in these two groups may therefore differ.

If trials do not report data for particular subgroups of participants then we will need to synthesise data at the level of the individual trial, where appropriate. We will identify studies as belonging to a particular subgroup if more than $2 / 3$ participants $(66 \%)$ belong to that category.

If trials present data for subgroups of individuals within the trial we will use this for subgroup analysis, where applicable, regardless of whether trials have stratified their randomisation according to those subgroups.

We anticipate that the varying methods used for olfactory training may be a source of heterogeneity in effects. If we identify heterogeneity in the comparison of olfactory training then we will explore this considering the following factors:

- classical versus modified olfactory training (using the same scents throughout compared to changing the scents);

- the duration of the intervention.

\section{Sensitivity analysis}

We plan to carry out sensitivity analyses to determine whether the findings are robust to the decisions made in the course of identifying, screening and analysing the trials. We plan to conduct sensitivity analysis for the following factors, whenever possible:

- impact of model chosen: to investigate whether the use of a random-effects model impacts on the effect estimates;

- inclusion of studies with concurrent treatments: to exclude these studies from the pooled estimates of effect for any intervention;
- method of COVID-19 diagnosis: to exclude studies where only a clinical method of COVID-19 diagnosis was used (rather than laboratory confirmed).

\section{Summary of findings and assessment of the certainty of the evidence}

Two independent authors will use the GRADE approach to rate the overall certainty of evidence using GRADEpro GDT (https:// gradepro.org/). The certainty of evidence reflects the extent to which we are confident that an estimate of effect is correct and we will apply this in the interpretation of results. There are four possible ratings: high, moderate, low and very low. A rating of high certainty of evidence implies that we are confident in our estimate of effect and that further research is very unlikely to change our confidence in the estimate of effect. A rating of very low certainty implies that any estimate of effect obtained is very uncertain.

The GRADE approach rates evidence from RCTs that do not have serious limitations as high certainty. However, several factors can lead to the downgrading of the evidence to moderate, low or very low. The degree of downgrading is determined by the seriousness of these factors:

- study limitations (risk of bias);

- inconsistency;

- indirectness of evidence;

- imprecision; and

- publication bias.

We will include a 'Summary of findings' table, constructed according to the recommendations described in Chapter 14 of the Cochrane Handbook for Systematic Reviews of Interventions (Handbook 2020), for the following comparison(s):

- intranasal steroid drops/rinses versus no treatment/placebo;

- intranasal steroid sprays versus no treatment/placebo;

- olfactory training versus no treatment/placebo;

- intranasal vitamin A versus no treatment/placebo.

We will include the following outcomes in the 'Summary of findings' tables:

- recovery of sense of smell (as reported by the participants);

- disease-related quality of life, as assessed by the Olfactory Disorders Questionnaire (or another validated questionnaire);

- serious adverse effects;

- change in sense of smell (as identified by psychophysical testing);

- overall, generic quality of life, as assessed by validated methods (e.g. EQ-5D);

- presence of parosmia;

- other adverse effects (including nosebleeds/bloody discharge).

\section{Methods for future updates}

\section{Living systematic review considerations}

We will review the scope and methods of this review approximately yearly (or more frequently if appropriate) in the light of potential changes in the topic area, or the evidence being included in the review (for example, additional comparisons, interventions or outcomes, or new review methods available). 
Conditions under which the review will no longer be maintained as a living systematic review

The review will no longer be maintained as a living systematic review once there is high-certainty evidence obtained for the primary effectiveness outcomes of the review; once new studies are not expected to be conducted regularly for the interventions included in this review; or once the review topic is no longer a priority for health care decision-making.

\section{A C K N OWLEDGEMENTS}

This study is one of a number of COVID-19 studies that have been funded by the National Institute for Health Research (NIHR) as part of its Recovery and Learning call, totalling $£ 5.5 \mathrm{~m}$ in funding, to help better manage current and future waves of the COVID-19 pandemic and investigate its long-term impacts on the health and care system beyond the acute phase.

This project was also supported by the National Institute for Health Research, via Cochrane Infrastructure, Cochrane Programme Grant or Cochrane Incentive funding to Cochrane ENT. The views and opinions expressed therein are those of the authors and do not necessarily reflect those of the Systematic Reviews Programme, NIHR, NHS or the Department of Health.

The authors would like to extend their sincere thanks to the board members of AbScent and Fifth Sense for their insights and significant contributions to the development of this protocol. We would also like to thank the many individuals affected by smell loss who took the time to complete our surveys, which were invaluable when prioritising outcomes for this review.

The authors would like to acknowledge Lee Yee Chong for her work on generic methods content, some of which has been adapted for use in this review (with permission).

The authors are grateful to Joanne Abbott, Information Specialist with the Cochrane Pain, Palliative and Supportive Care Group, for providing peer review comments on the draft search methods. 


\section{RE F E R E N C E S}

\section{Additional references}

\section{Alexander 2006}

Alexander TH, Davidson TM. Intranasal zinc and anosmia: the zinc-induced anosmia syndrome. Laryngoscope 2006;116(2):217-20. [DOI: 10.1097/01.mlg.0000191549.17796.13] [PMID: 16467707]

\section{Bigman 2020}

Bigman G. Age-related smell and taste impairments and vitamin D associations in the U.S. Adults National Health and Nutrition Examination Survey. Nutrients 2020;12(4):984. [DOI: 10.3390/ nu12040984] [PMID: 32252288]

\section{Bilinska 2020}

Bilinska K, Butowt R. Anosmia in COVID-19: a bumpy road to establishing a cellular mechanism. ACS Chemical Neuroscience 2020;11(15):2152-5.

\section{Boscolo-Rizzo 2020}

Boscolo-Rizzo P, Borsetto D, Fabbris C, Spinato G, Frezza D, Menegaldo A, et al. Evolution of altered sense of smell or taste in patients with mildly symptomatic COVID-19. JAMA Otolaryngology--Head \& Neck Surgery 2020;146(8):729-32. [PMID: 32614442]

\section{Butowt 2020}

Butowt R, von Bartheld CS. Anosmia in COVID-19: underlying mechanisms and assessment of an olfactory route to brain infection. Neuroscientist 2020 Sep 11 [Epub ahead of print]. [DOI: $10.1177 / 1073858420956905]$ [PMID: 32914699]

\section{Cain 1988}

Cain WS, Gent JF, Goodspeed RB, Leonard G. Evaluation of olfactory dysfunction in the Connecticut Chemosensory Clinical Research Center. Laryngoscope 1988;98(1):83-8. [PMID: 3336267]

\section{Chary 2020}

Chary E, Carsuzaa F, Trijolet J-P, Capitaine A-L, RoncatoSaberan M, Fouet K, et al. Prevalence and recovery from olfactory and gustatory dysfunctions in COVID-19 infection: a prospective multicenter study. American Journal of Rhinology \& Allergy 2020;34(5):686-93. [DOI: 10.1177/1945892420930954]

\section{Croy 2014}

Croy I, Nordin S, Hummel T. Olfactory disorders and quality of life--an updated review. Chemical Senses 2014;39(3):185-94. [DOI: 10.1093/chemse/bjt072]

\section{Dai 2016}

Dai Q, Pang Z, Yu H. Recovery of olfactory function in postviral olfactory dysfunction patients after acupuncture treatment. Evidence-based Complementary and Alternative Medicine: eCAM 2016;2016:4986034. [PMID: 27034689]

\section{Damm 2004}

Damm M, Temmel A, Welge-Lüssen A, Eckel HE, Kreft MP, Klussmann JP, et al. Olfactory dysfunctions. Epidemiology and therapy in Germany, Austria and Switzerland [Riechstörungen. Epidemiologie und Therapie in Deutschland, Osterreich und der Schweiz]. HNO 2004;52(2):112-20. [PMID: 14968312]

\section{Derin 2016}

Derin S, Koseoglu S, Sahin C, Sahan M. Effect of vitamin B12 deficiency on olfactory function. International Forum of Allergy \& Rhinology 2016;6(10):1051-5. [PMID: 27119316]

\section{Egger 1997}

Egger M, Davey Smith G, Schneider M, Minder C. Bias in meta-analysis detected by a simple, graphical test. $B M J$ 1997;315(7109):629.

\section{Erskine 2020}

Erskine SE, Philpott CM. An unmet need: patients with smell and taste disorders. Clinical Otolaryngology 2020;45(2):197-203.

\section{Fuccillo 2020}

Fuccillo E, Saibene AM, Canevini MP, Felisati G. Olfactory disorders in coronavirus disease 2019 patients: a systematic literature review. Journal of Laryngology \& Otology 2020 Sep 15 [Epub ahead of print]. [DOI: 10.1017/S0022215120002005]

\section{Gerkin 2020}

Gerkin RC, Ohla K, Veldhuizen MG, Joseph PV, Kelly CE, Bakkeet AJ, et al. The best COVID-19 predictor is recent smell loss: a cross-sectional study. https://www.medrxiv.org/ content/10.1101/2020.07.22.20157263v2 2020. [DOI: $10.1101 / 2020.07 .22 .20157263]$

\section{Gudziol 2006}

Gudziol V, Lötsch J, Hähner A, Zahnert T, Hummel T. Clinical significance of results from olfactory testing. Laryngoscope 2006;116(10):1858-63. [PMID: 17003712]

\section{Handbook 2011}

Higgins JPT, Green S (editors). Cochrane Handbook for Systematic Reviews of Interventions Version 5.1.0 [updated March 2011]. The Cochrane Collaboration, 2011. Available from www.cochrane-handbook.org.

\section{Handbook 2020}

Higgins JPT, Thomas J, Chandler J, Cumpston M, Li T, Page MJ, Welch VA (editors). Cochrane Handbook for Systematic Reviews of Interventions version 6.1 (updated September 2020). Available from www.training.cochrane.org/handbook: Cochrane, 2020.

\section{Hopkins 2020a}

Hopkins C, Kumar N. Loss of sense of smell as a marker of COVID-19 infection. https://www.sforl.org/wp-content/ uploads/2020/03/Loss-of-sense-of-smell-as-marker-ofCOVID-21-Mar-2020.pdf.

\section{Hopkins 2020b}

Hopkins C, Burges Watson DL, Kelly C, Deary V, Smith BC. Managing long COVID: don't overlook olfactory dysfunction. BMJ 2020;370:m3736. 


\section{Hopkins 2020c}

Hopkins C, Lechien JR, Saussez S. More that ACE2? NRP1 may play a central role in the underlying pathophysiological mechanism of olfactory dysfunction in COVID-19 and its association with enhanced survival. Medical Hypotheses 2020 Nov 20 [Epub ahead of print]. [DOI: 10.1016/ j.mehy.2020.110406]

\section{Huart 2020}

Huart C, Philpott C, Konstantinidis I, Altundag A, Whitcroft KL, Trecca EMC, et al. Comparison of COVID-19 and common cold chemosensory dysfunction. Rhinology 2020;58(6):623-5. [DOI: 10.4193/Rhin20.251] [PMID: 32812014]

\section{Hummel 2002}

Hummel T, Heilmann S, Hüttenbriuk KB. Lipoic acid in the treatment of smell dysfunction following viral infection of the upper respiratory tract. Laryngoscope 2002;112(11):2076-80. [PMID: 12439184]

\section{Hummel 2016}

Hummel T, Whitcroft KL, Andrews P, Altundag A, Cinghi C, Costanzo RM, et al. Position paper on olfactory dysfunction. Rhinology 2016;56(1):1-30. [PMID: 28623665]

\section{Hummel 2017}

Hummel T, Whitcroft KL, Rueter G, Haehner A. Intranasal vitamin $A$ is beneficial in post-infectious olfactory loss. European Archives of Oto-rhino-laryngology 2017;274(7):2819-25. [PMID: 28434127]

\section{Klopfenstein 2020}

Klopfenstein T, Kadiane-Oussou NJ, Toko L, Royer PY, Lepiller Q, Gendrin V, et al. Features of anosmia in COVID-19. Medecine et Maladies Infectieuses 2020;50(5):436-9. [DOI: 10.1016/ j.medmal.2020.04.006]

\section{Landis 2003}

Landis BN, Hummel T, Hugentobler M, Giger R, Lacroix JS. Ratings of overall olfactory function. Chemical Senses 2003;28(8):691-4. [DOI: 10.1093/chemse/bjg061] [PMID: $14627537]$

\section{Lechien 2020}

Lechien JR, Chiesa-Estomba CM, De Siati DR, Horoi M, Le Bon SD, Rodriguez A, et al. Olfactory and gustatory dysfunctions as a clinical presentation of mild-to-moderate forms of the coronavirus disease (COVID-19): a multicenter European study. European Archives of Oto-rhino-laryngology 2020;277(8):2251-61. [DOI: 10.1007/s00405-020-05965-1]

\section{Lefebvre 2020}

Lefebvre C, Glanville J, Briscoe S, Littlewood A, Marshall C, Metzendorf M-I, et al. Technical Supplement to Chapter 4: Searching for and selecting studies. In: Higgins JPT, Thomas J, Chandler J, Cumpston MS, Li T, Page MJ, Welch VA, editors(s). Cochrane Handbook for Systematic Reviews of Interventions Version 6.1 (updated September 2020). Cochrane, 2020.

\section{Mao 2020}

Mao L, Jin H, Wang M, Hu Y, Chen S, He Q, et al. Neurologic manifestations of hospitalized patients with coronavirus disease 2019 in Wuhan, China. JAMA Neurology 2020;77(6):683-90.

\section{Marshall 2018}

Marshall J, Noel-Storr AH, Kuiper J, Thomas J, Wallace BC. Machine learning for identifying randomized controlled trials: an evaluation and practitioner's guide. Research Synthesis Methods 2018;9(4):602-14.

\section{Martineau 2020}

Martineau AR, Forouhi NG. Vitamin D for COVID-19: a case to answer? Lancet. Diabetes \& Endocrinology 2020;8(9):735-6. [PMID: 32758429]

\section{Mattos 2018}

Mattos JL, Schlosser RJ, Mace JC, Smith TL, Soler ZM. Establishing the minimal clinically important difference for the Questionnaire of Olfactory Disorders. International Forum of Allergy \& Rhinology 2018;8(9):1041-6. [PMID: 29719139]

\section{McDonald 2017}

McDonald S, Noel-Storr AH, Thomas J. Harnessing the efficiencies of machine learning and Cochrane Crowd to identify randomised trials for individual Cochrane reviews. In: Global Evidence Summit; 2017 Sep 13-17; Cape Town, South Africa. 2017.

\section{Meng 2020}

Meng X, Deng Y, Dai Z, Meng Z. COVID-19 and anosmia: a review based on up-to-date knowledge. American Journal of Otolaryngology 2020;41(5):102581.

\section{Menni 2020}

Menni C, Valdes AM, Freidin MB, Sudre CH, Nguyen LH, Drew DA, et al. Real-time tracking of self-reported symptoms to predict potential COVID-19. Nature Medicine 2020;26(7):1037-40. [DOI: 10.1038/s41591-020-0916-2]

\section{Noel-Storr 2018}

Noel-Storr AH. The Project Transform Team. Cochrane Crowd: new ways of working together to produce health evidence. In: Evidence Live; 2018 Jun 18-20; Oxford, UK. 2018.

\section{Parma 2020}

Parma V, Ohla K, Veldhuizen MG, Niv MY, Kelly CE, Bakke AJ, et al. More than smell - COVID-19 is associated with severe impairment of smell, taste and chemesthesis. Chemical Senses 2020;45(7):609-22. [DOI: 10.1093/chemse/bjaa041]

\section{Pekala 2016}

Pekala K, Chandra RK, Turner JH. Efficacy of olfactory training in patients with olfactory loss: a systematic review and meta-analysis. International Forum of Allergy \& Rhinology 2016;6(3):299-307.

\section{Philpott 2014}

Philpott CM, Boak D. The impact of olfactory disorders in the United Kingdom. Chemical Senses 2014;39(8):711-8. 


\section{Quint 2002}

Quint C, Temmel AF, Hummel T, Ehrenberger K. The quinoxaline derivative caroverine in the treatment of sensorineural smell disorders: a proof-of-concept study. Acta Oto-laryngologica 2002;122(8):877-81. [PMID: 12542209]

\section{Reden 2011}

Reden J, Herting B, Lill K, Kern R, Hummel T. Treatment of postinfectious olfactory disorders with minocycline: a double-blind, placebo-controlled study. Laryngoscope 2011;121(3):679-82. [PMID: 21287560]

\section{RevMan 2020 [Computer program]}

The Cochrane Collaboration Review Manager 5 (RevMan 5). Version 5.4. Copenhagen: The Cochrane Collaboration, 2020.

\section{Rocke 2020}

Rocke J, Hopkins C, Philpott C, Kumar N. Is loss of sense of smell a diagnostic marker in COVID-19: a systematic review and metaanalysis. Clinical Otolaryngology 2020;45(6):914-22.

\section{Saussez 2020}

Saussez S, Lechien JR, Hopkins C. Anosmia: an evolution of our understanding of its importance in COVID-19 and what questions remain to be answered. European Archives of Otorhino-laryngology 2020 Sep 9 [Epub ahead of print]. [DOI: 10.1007/s00405-020-06285-0] [PMID: 32909060]

\section{Seiden 2001}

Seiden AM, Duncan HJ. The diagnosis of a conductive olfactory loss. Laryngoscope 2001;111(1):9-14.

\section{Sorokowska 2017}

Sorokowska A, Drechsler E, Karwowski M, Hummel T. Effects of olfactory training: a meta-analysis. Rhinology 2017;55(1):17-26. [DOI: 10.4193/Rhin16.195] [PMID: 28040824]

\section{Spinato 2020}

Spinato G, Fabbris C, Polesel J, Cazzador D, Borsetto D, Hopkins $C$, et al. Alterations in smell or taste in mildly symptomatic outpatients with SARS-CoV-2 infection. JAMA 2020;323(20):2089-90.

\section{Thomas 2017}

Thomas J, Noel-Storr AH, Marshall I, Wallace B, McDonald S, Mavergames C, et al, Living Systematic Review Network. Living systematic reviews 2: combining human and machine effort. Journal of Clinical Epidemiology 2017;91:31-7.

\section{Tierney 2007}

Tierney JF, Stewart LA, Ghersi D, Burdett S, Sydes MR. Practical methods for incorporating summary time-to-event data into meta-analysis. Trials 2007;8:16.

\section{Vent 2010}

Vent J, Wang DW, Damm M. Effects of traditional Chinese acupuncture in post-viral olfactory dysfunction. Otolaryngology - Head and Neck Surgery 2010;142(4):505-9. [PMID: 20304268]

\section{von Bartheld 2020}

von Bartheld CS, Hagen MM, Butowt R. Prevalence of chemosensory dysfunction in COVID-19 patients: a systematic review and meta-analysis reveals significant ethnic differences. ACS Chemical Neuroscience 2020;11(19):2944-61. [DOI: 10.1021/ acschemneuro.0c00460]

\section{Wallace 2017}

Wallace BC, Noel-Storr AH, Marshall IJ, Cohen AM, Smalheiser NR, et al. Identifying reports of randomized controlled trials (RCTs) via a hybrid machine learning and crowdsourcing approach. Journal of the American Medical Informatics Association 2017;24(6):1165-8.

\section{Webster 2021}

Webster KE, Mackeith S, Philpott C, Hopkins C, Burton MJ. Interventions for the prevention of persistent post-COVID-19 olfactory dysfunction. Cochrane Database of Systematic Reviews 2021, Issue 2.

\section{Welge-Luessen 2005}

Welge-Luessen A, Hummel T, Stojan T, Wolfensberger M. What is the correlation between ratings and measures of olfactory function in patients with olfactory loss? American Journal of Rhinology 2005;19(6):567-71. [DOI: $10.1177 / 194589240501900606]$

\section{AP P E N DICES}

\section{Appendix 1. Draft search strategies}

\section{CENTRAL (CRS)}

1 (("2019 nCoV" or 2019nCoV or "COVID 19" or COVID19 or "new coronavirus" or "novel coronavirus" or "novel corona virus" or "SARS CoV-2" or SARS-CoV2 or SARSCoV2 or "2019-novel CoV" or ncov19 or ncov-19 or nCov 2019 or COVID-19 or SARSCoV-2 or SARS(OV-2)):AB,EH,KW,KY,MC,MH,TI,TO AND CENTRAL:TARGET 2844

2 ((Wuhan and (coronavirus or "corona virus"))):AB,EH,KW,KY,MC,MH,TI,TO AND CENTRAL:TARGET 124

3 (((coronavirus or "corona virus" or COVID) adj3 "2019")):AB,EH,KW,KY,MC,MH,TI,TO AND CENTRAL:TARGET 582

4 ((wuhan adj2 (disease or virus))):AB,EH,KW,KY,MC,MH,TI,TO AND CENTRAL:TARGET 4

5 ((coronavirus or "corona virus" or COVID) ):AB,EH,KW,KY,MC,MH,TI,TO AND CENTRAL:TARGET 2919

6 (2020 or 2021):YR AND CENTRAL:TARGET 63399 
7 \#5 AND \#6 AND CENTRAL:TARGET 2801

8 \#1 OR \#2 OR \#3 OR \#4 OR \#7 AND CENTRAL:TARGET 2877

9 MESH DESCRIPTOR Olfaction Disorders EXPLODE ALL AND CENTRAL:TARGET 118

10 (Olfaction or olfactory or Dysosmia* or Paraosmia* or Anosmia* or hyposmia* or phantosmia* or Cacosmia* or microsmia*):AB,EH,KW,KY,MC,MH,TI,TO AND CENTRAL:TARGET 1201

11 (smell $^{\star}$ adj6 (disorder ${ }^{\star}$ or loss or distort* or alter* or dsyfunction or impair ${ }^{\star}$ or abscen* or reduce* or different ${ }^{\star}$ or sensation* or abnormal $^{\star}$ or perception* or change* or expected or decreas* or deficit*)):AB,EH,KW,KY,MC,MH,TI,TO AND CENTRAL:TARGET 424

12 (smell* adj6 (prevent* or rehab* or recover* or therap* or train* or retrain*)):AB,EH,KW,KY,MC,MH,TI,TO AND CENTRAL:TARGET 151

13 \#9 OR \#10 OR \#11 OR \#12 AND CENTRAL:TARGET 1474

\section{4 \#13 AND \#8 AND CENTRAL:TARGET 34}

\section{MEDLINE (Ovid)}

1 ("2019 nCoV" or 2019nCoV or "COVID 19" or COVID19 or "new coronavirus" or "novel coronavirus" or "novel corona virus" or "SARS CoV-2" or SARS-CoV2 or SARSCoV2 or "2019-novel CoV" or ncov19 or ncov-19 or nCov 2019 or COVID-19 or SARSCoV-2 or SARS-CoV-2).ab,ti. 64357

2 (Wuhan and (coronavirus or "corona virus")).ab,ti. 2686

3 (coronavirus or "corona virus" or COVID) adj3 "2019").ab,ti. 14622

4 (wuhan adj2 (disease or virus)).ab,ti. 69

5 ("LAMP assay" or "COVID-19" or "COVID-19 drug treatment" or "COVID-19 diagnostic testing" or "CoVID-19 serotherapy" or "COVID-19 vaccine" or "severe acute respiratory syndrome coronavirus 2" or "spike protein, SARS-CoV-2").os. 27706

6 (coronavirus or "corona virus" or COVID).ab,ti. 73711

7 limit 6 to yr="2020 -Current" 64172

81 or 2 or 3 or 4 or 5 or 768776

9 exp olfaction disorders/ 4393

10 (Olfaction or olfactory or Dysosmia* or Paraosmia* or Anosmia* or hyposmia* or phantosmia* or Cacosmia* or microsmia*).ab,ti. 49706

11 (smell $^{\star}$ adj6 (disorder ${ }^{\star}$ or loss or distort* or alter ${ }^{\star}$ or dsyfunction or impair ${ }^{\star}$ or abscen ${ }^{\star}$ or reduce or different $^{\star}$ or sensation* or abnormal $^{\star}$ or perception ${ }^{\star}$ or change* or expected or decreas ${ }^{\star}$ or deficit $\left.\left.{ }^{\star}\right)\right) \cdot$ ab,ti. 2401

$12\left(\right.$ smell $^{\star}$ adj6 (prevent* or rehab* $^{\star}$ or recover $^{\star}$ or therap ${ }^{\star}$ or train* or retrain $\left.{ }^{\star}\right)$ ).ab,ti. 180

139 or 10 or 11 or 1251681

148 and 13768

15 randomized controlled trial.pt. 516072

16 controlled clinical trial.pt. 93905

17 randomized.ab. 496684

18 placebo.ab. 212020

19 drug therapy.fs. 2246822

20 randomly.ab. 343595

21 trial.ab. 525027

22 groups.ab. 2108399

2315 or 16 or 17 or 18 or 19 or 20 or 21 or 224824610 
24 exp animals/ not humans.sh. 4750713

2523 not 244189853

2614 and 2572

Cochrane COVID-19 Study Register (CRS)

1 MESH DESCRIPTOR Olfaction Disorders EXPLODE ALL AND COVID19:INREGISTER 5

2 (Olfaction or olfactory or Dysosmia* or Paraosmia* or Anosmia* or hyposmia* or phantosmia* or Cacosmia* or microsmia*) AND COVID19:INREGISTER 392

3 (smell ${ }^{\star}$ adj6 (disorder ${ }^{\star}$ or loss or distort* or alter $^{\star}$ or dsyfunction or impair ${ }^{\star}$ or abscen ${ }^{\star}$ or reduce or different $^{\star}$ or sensation $^{\star}$ or abnormal $^{\star}$ or perception* or change* or expected or decreas* or deficit*)) AND COVID19:INREGISTER 180

4 (smell* adj6 (prevent* or rehab* or recover* or therap* or train* or retrain $\left.{ }^{\star}\right)$ ) AND COVID19:INREGISTER 17

5 \#1 OR \#2 OR \#3 OR \#4 483

6 (interventional):SY AND COVID19:INREGISTER 3460

7 \#6 AND \#5 37

\section{H I S T O R Y}

Protocol first published: Issue 2, 2021

\section{CONTRIBUTIONSOF AUTHORS}

Katie Webster: scoped, designed and drafted the protocol with the help of the other authors.

Samuel MacKeith: clinical guidance at all stages of project scoping and protocol development; commented on and edited the draft protocol, and agreed the final version.

Carl Philpott: clinical guidance at all stages of project scoping and protocol development; commented on and edited the draft protocol, and agreed the final version.

Claire Hopkins: clinical guidance at all stages of project scoping and protocol development; commented on and edited the draft protocol, and agreed the final version.

Martin Burton: clinical guidance at all stages of project scoping and protocol development; commented on and edited the draft protocol, and agreed the final version.

\section{DECLARATIONS OF INTEREST}

Katie Webster: none known.

Samuel MacKeith: Sam MacKeith is Assistant Co-ordinating Editor of Cochrane ENT, but had no role in the editorial process for this review. Carl Philpott: Professor Carl Philpott sees and treats patients with COVID-19 related smell loss. He has written various online publications on the topic. He is a Trustee for the charity Fifth Sense.

Claire Hopkins: Professor Claire Hopkins sees and treats patients with COVID-19 related smell loss. She has spoken on the association between COVID and smell loss in multiple media outlets. She is senior author of the British Rhinological Society position paper on management of COVID-19 related smell loss.

Martin Burton: Professor Martin Burton is joint Co-ordinating Editor of Cochrane ENT, but had no role in the editorial process for this review.

\section{SOURCES OF SUPPORT}

\section{Internal sources}

- No sources of support supplied

\section{External sources}

- National Institute for Health Research, UK Infrastructure funding for Cochrane ENT

- National Institute for Health Research (NIHR) COVID-19: Recovery and Learning programme, UK

Award NIHR132103 